\title{
COMPUTATIONAL RESULTS FOR INTEGRAL EQUATIONS IN SPACE-TIME
}

\author{
LECHOSŁAW HĄCIA \\ Institute of Mathematics, Poznań University of Technology \\ Piotrowo 3A, 60-965 Poznań, Poland \\ e-mail address: Ihacia@math.put.poznan.pl \\ (Received 13 September 2001)
}

\begin{abstract}
An important class of integral equations in space-time is discussed. The studied equations, called Volterra-Fredholm integral equations or integral equations of the mixed type are used in technology, mechanics, biology and the heat conduction theory. First, the Galerkin method is applied to the Fredholm integral equations in space-time. Next, it is used for the Volterra-Fredholm integral equations. The presented algorithm is illustrated by graphs and tables.
\end{abstract}

\section{INTRODUCTION}

Consider the following integral equation

$$
f(x, t)=g(x, t)+\int_{0}^{T} \int_{M} N(x, t, y, s) f(y, s) d y d s,
$$

where $M$ is a compact subset of Euclidean space $R^{k}$ or compact manifold. Suppose that this equation has a unique solution in the space $L^{2}(D)$

$$
D=M \times[0, T], \quad \text { for } \quad g \in L^{2}(D) \quad \text { and } \quad N \in L^{2}(D \times D) .
$$

The considered integral equations in space-time play very important role in mechanics and technology. Presented equations arise in the wave theory. Some initial-boundary problems for a number of differential partial equations in physics can be reduced to the considered integral equations.

\section{GALERKIN METHOD}

We search for a numerical solution in the form

$$
f_{n}(x, t)=\sum_{j=1}^{n} c_{j} \Phi_{j}(x, t)
$$

where $\Phi_{j}$ is an orthonormal basis in $L^{2}(D)$ and constants $c_{1}, c_{2}, \ldots, c_{n}$ are choosen such that 


$$
\left(\varepsilon_{n}, \Phi_{j}\right)=0, \quad j=1,2, \ldots, n,
$$

and the deviation function is defined in the following way

$$
\varepsilon_{n}(x, t)=f_{n}(x, t)-\int_{0}^{T} \int_{M} N(x, t, y, s) f_{n}(y, s) d y d s-g(x, t) .
$$

Then we get

$$
\begin{aligned}
\int_{0}^{T} \int_{M} f_{n}(x, t) \Phi_{j}(x, t) d x d t & -\int_{0}^{T} \int_{M}\left(\int_{0}^{T} \int_{M} N(x, t, y, s) f_{n}(y, s) d y d s\right) \Phi_{j}(x, t) d x d t= \\
& =\int_{0}^{T} \int_{M} g(x, t) \Phi_{j}(x, t) d x d t .
\end{aligned}
$$

Putting (2) in the above equality and taking into account the orthonormality of the system $\left(\Phi_{n}\right)$ we obtain the following system of algebraic equations

$$
c_{j}-\sum_{k=1}^{n} c_{k} N_{k j}=g_{j}, \quad j=1,2, \ldots, n,
$$

where

$$
\begin{gathered}
N_{k j}=\int_{0}^{T} \int_{M}\left(\int_{0}^{T} \int_{M} N(x, t, y, s) \Phi_{k}(y, s) d y d s\right) \Phi_{j}(x, t) d x d t \quad k=1,2, \ldots n \quad j=1,2, \ldots n \\
g_{j}=\int_{0}^{T} \int_{M} g(x, t) \Phi_{j}(x, t) d x d t \quad j=1,2, \ldots n .
\end{gathered}
$$

In the case $N(x, t, y, s)=0, t \leq s \leq T$ equation (1) is reduced to the equation

$$
f(x, t)=g(x, t)+\int_{0}^{t} \int_{M} N(x, t, y, s) f(y, s) d y d s,
$$

so-called Volterra-Fredholm integral equation or a mixed integral equation in the space-time.

Considered integral equations of the Volterra-Fredholm type are very important in mechanics and biology. They arise in the heat conduction theory and the mathematical modelling of the spatio-temporal development of an epidemic. The spread of the disease in the given population can be described by the Volterra-Fredholm integral equations.

The general theoty of these equations was presented in weighted spaces [3]. It has been shown that the integral equation (4) has a unique solution in spaces $\mathrm{C}$ and $L^{p}(p \geq 1)$. Numerical 
solutions were investigated in [1-6], In this paper the Galerkin method is used for solving the equation (4) and it leads to the system of algebraic equations (3) with

$$
\begin{gathered}
N_{k j}=\int_{0}^{T} \int_{M}\left(\int_{0} \int_{M} N(x, t, y, s) \Phi_{k}(y, s) d y d s\right) \Phi_{j}(x, t) d x d t \\
k=1,2, \ldots, n \quad j=1,2, \ldots, n
\end{gathered}
$$

where

$$
\Phi_{j}(x, t)=u_{p}(x) v_{q}(t)=u(p, x) \cdot v(q, t)
$$

Our considerations are restricted to the orthonormal Legendre polynomials defined in intervals $[-1,1]$ and $[0,1]$, respectively.

\section{IMPLEMENTATION IN MAPLE V}

$$
\begin{aligned}
& A:=p \rightarrow \int_{-1}^{1} u(p, x)^{2} d x \\
& B:=q \rightarrow \int_{0}^{1} v(q, t)^{2} d t \\
& q_{n}:=(p, q) \rightarrow \int_{0}^{1} \int_{-1}^{1} g(x, t) u(p, x) v(q, t) d x d t \\
& V q_{n}:=\operatorname{vector}(n \times n) \\
& \text { for } p 1 . . n \\
& \text { for } q 1 . . n \\
& V q_{n}[n(p-1)+q]:=-q_{n}(p, q) \\
& \text { end } \\
& \text { end } \\
& o p\left(V q_{n}\right) \\
& N_{n}:=(i, j, p, q) \rightarrow \int_{0}^{1} \int_{-1}^{1}\left[\int_{0}^{t} \int_{-1}^{1} N(x, t, y, s) u(i, y) v(j, s)\right] d y d s \cdot u(p, x) v(q, t) d x d t
\end{aligned}
$$

Values of integrals $N_{n}$

$$
\begin{aligned}
& M N_{n}:=\operatorname{matrix}(n \times n, n \times n) \\
& \text { for } p 1 . . n
\end{aligned}
$$


for $q 1 . . n$

row $:=n(p-1)+q$

for $i 1 . . n$

for $j 1 . . n$

$M N_{n}[$ row, $n(i-1)+j]:=N_{n}(i, j, p, q)$ end end

$M N_{n}[$ row, row $]:=M N_{n}[$ row, row $] \backslash-A(p) B(q)$ end end

for $i$ to $n$

for $j$ to $n$

$c_{i j}:=\operatorname{solve}[n(i-I)+j]$ od od

$o p(c)$

$$
f d:=(x, t) \rightarrow \sum_{j=1}^{n}\left(\sum_{i=1}^{n} c_{i j} u(i, x)\right) v(j, t)
$$

\section{Example 1}

$$
\begin{aligned}
f(x, t)=x \sin (t)+ & \frac{1}{3} x^{2} t^{2} \mathrm{e}^{t} \cos (t)-\frac{1}{3} x^{2} t^{2} \mathrm{e}^{t} \sin (t)-\frac{1}{3} x^{2} t^{2}+ \\
& +\int_{0}^{t} \int_{-1}^{1} y x^{2} t^{2} e^{s} f(y, s) d y d s
\end{aligned}
$$

with

$$
f(x, t)=x \sin (t)
$$

Absolute errors:

$\underline{n=3}$

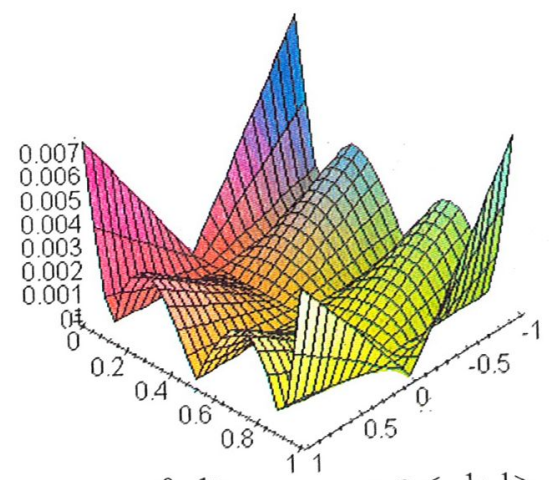

$t \in<0 ; 1>$

$x \in\langle-1 ; 1>$

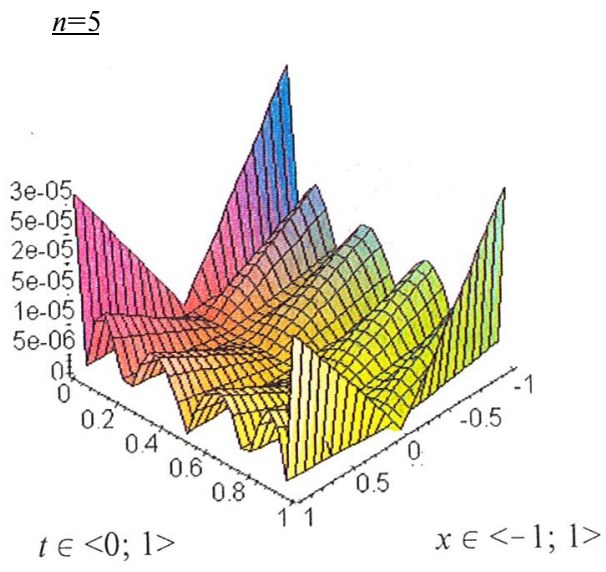




\section{Example 2}

$$
f(x, t)=x+\sin (t)-\frac{2}{3} e^{t}+\frac{2}{3}+\int_{0}^{t} \int_{-1}^{1} y e^{s} f(y, s) d y d s
$$

with an exact solution $f(x, t)=x+\sin (t)$
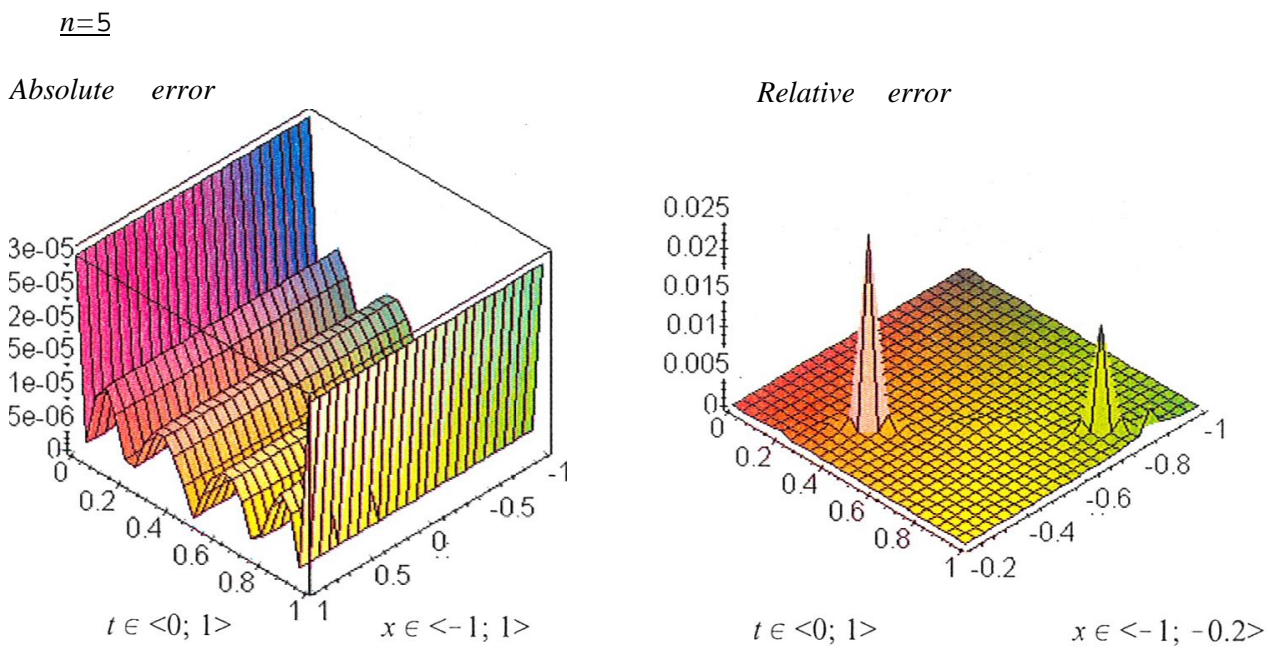

\section{$\underline{n=10}$}

Absolute error
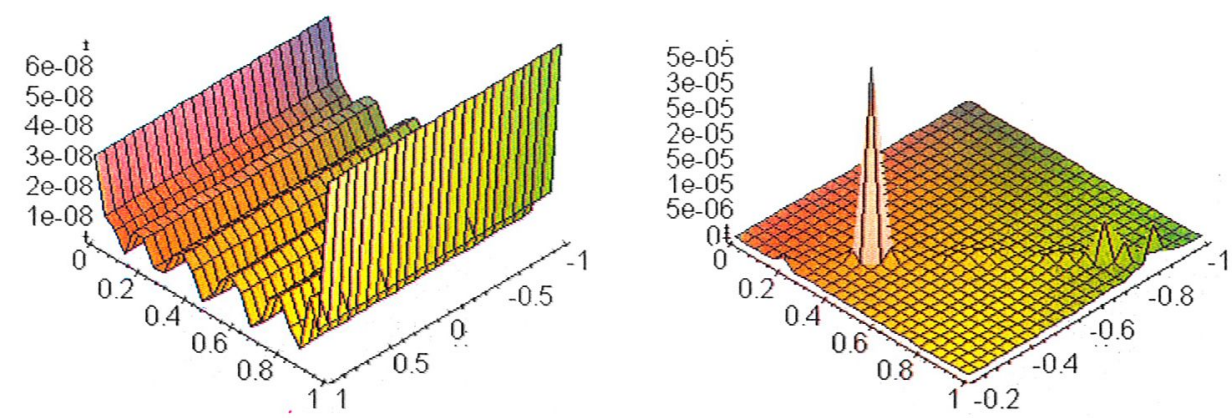

$t \in<0 ; 1>$

$x \in<-1 ; 1>$

$t \in<0 ; 1>$

$x \in<-1 ;-0.2>$ 
Example 3

$$
f(x, t)=e^{-t} x^{2}-\frac{2}{3} t^{3} x^{2}+\int_{0}^{t} \int_{-1}^{1} x^{2} t^{2} e^{s} f(y, s) d y d s
$$

where $f(x, t)=e^{-t} x^{2}$ is an exact solution.
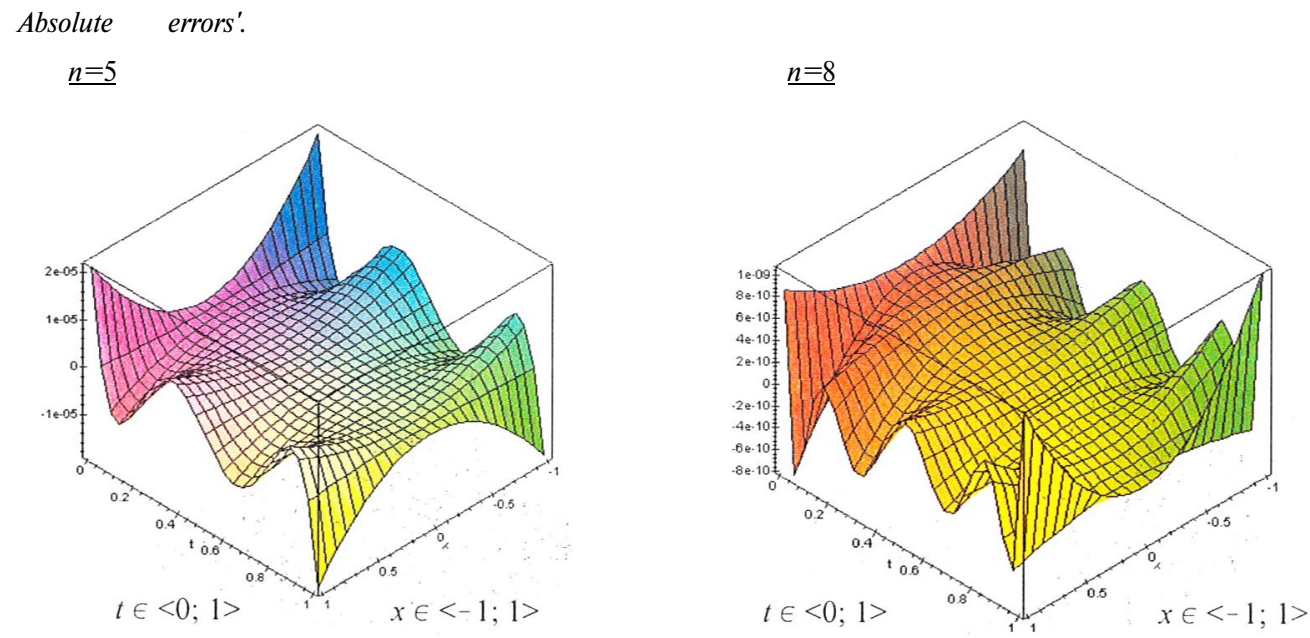

\section{Example 4}

$$
f(x, t)=\mathrm{e}^{-t} \cos x-\frac{1}{2} t \mathrm{e}^{-i} \cos x \cdot(2+\sin 2)+\int_{0}^{t} \int_{-1}^{1} \mathrm{e}^{s-t} \cos (x-y) f(y, s) d y d s
$$

where $f(x, t)=\mathrm{e}^{-t} \cos \mathrm{x}$ is an exact solution.

$\underline{n=7}$

Absolute error

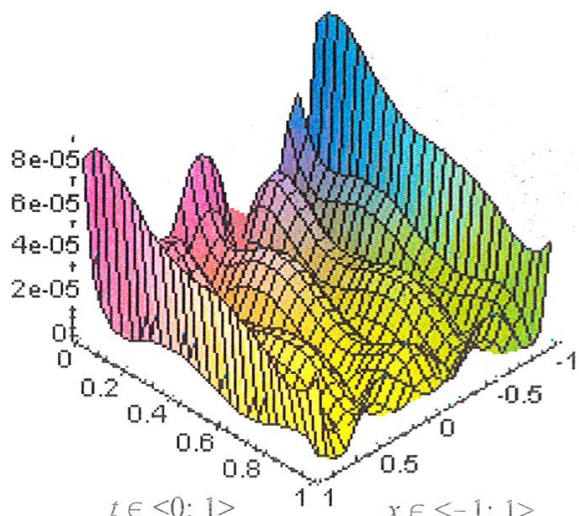

Relative error

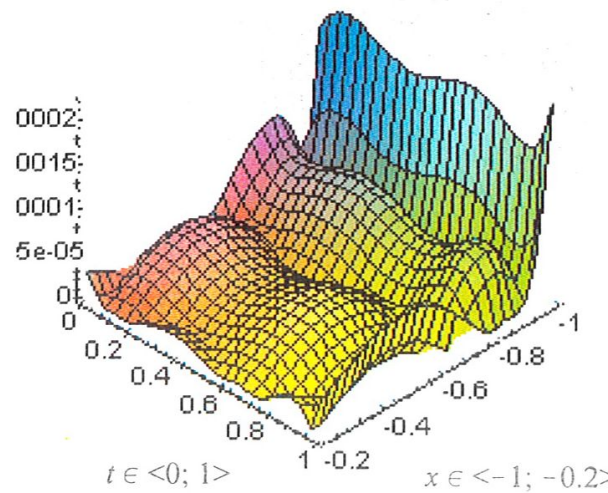




\section{$\underline{n=8}$}

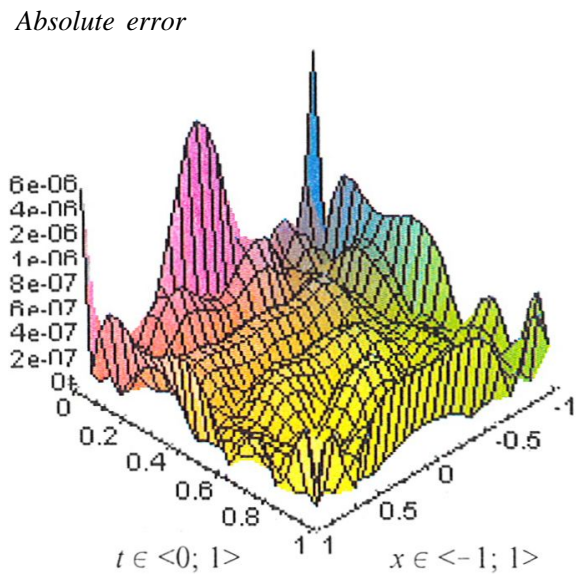

Relative error

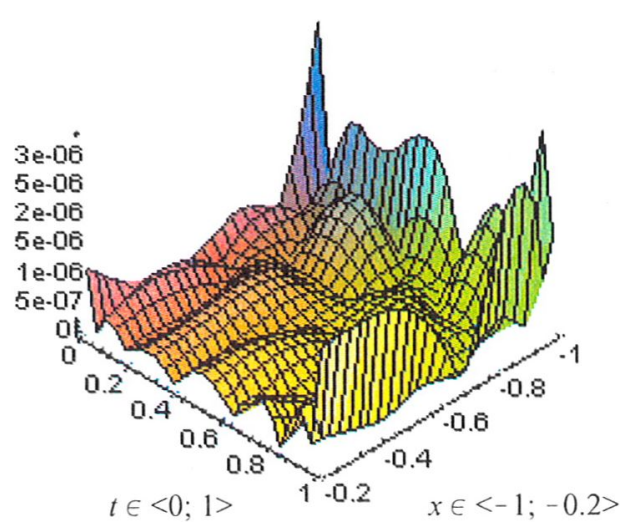

The exact analysis of the Galerkin method is presented below.

\section{Example 5}

$N:=(x, t, y, s) \rightarrow \exp (-y) * x^{\wedge} 2:$

$f:=(x, t)->\exp (x) * t^{\wedge} 2:$

$g:=f(x, t)-\operatorname{int}(\operatorname{int}(N(x, t, y, s) * f(y, s), y=-1 . .1), s=0 . . t):$

$g:=(x, t) \rightarrow f(x, t)-\operatorname{int}(\operatorname{int}(N(x, t, y, s) * f(y, s), y=-1 . .1), s=0 . . t):$

$u:=(k, x) \rightarrow P(k-1, x): v:=(l, t) \rightarrow P(l-1,2 * t-l):$

$n:=6$ :

dig: $:=30:$ digits $:=30:$

$A 0:=p->\operatorname{evalf}\left(\operatorname{int}\left((u(p, x))^{\wedge} 2, x=-1 . .1\right), \operatorname{dig}\right): B 0:=q-\operatorname{evalf}\left(\operatorname{int}\left((v(q, t))^{\wedge} 2, t=0 . .1\right), \operatorname{dig}\right):$

$g n:=(p, q)->\operatorname{evalf}(\operatorname{int}(\operatorname{int}(g(x, t) * u(p, x) * v(q, t), x=-. .1), t=0 . .1), \operatorname{dig}):$

with(linalg):

$\operatorname{Vgn}:=\operatorname{vector}\left(n^{*} n\right)$ :

for $p$ from 1 to $n$ do

for $q$ from 1 to $n$ do

$\operatorname{Vgn}\left[n^{*}(p-1)+q\right]:=-\operatorname{evalf}(g n(p, q))$ od: od:

$o p(\operatorname{Vg} n)$ :

$N n:=(i, j, p, q)-\operatorname{evalf(int}(\operatorname{int}(\operatorname{int}(i n t(N(x, t, y, s) * u(i, y) * v(j, s), y=-1 . .1), s=0 . . t) * u(p, x) * v(q, t), x=-1 \ldots 1)$, $t=0.1), \operatorname{dig})$ :

Mnn: $=\operatorname{matrix}\left(n^{*} n, n^{*} n\right)$ :

for $p$ from 1 to $n$ do

for $q$ from $I$ to $n$ do

row $:=(p-1) * n+q$;

for i from 1 to $n$ do

for $j$ from 1 to $n$ do

Mnn[row, $\left.(i-1){ }^{*} n+j\right]:=N n(i, j, p q) ;$ od; od; 
Mnn $[$ row , row $]:=\operatorname{Mnn}[$ row, row $]-A O(p) * B O(q) ;$ od;o d;

$o p(M N n)$ :

lns : = linsolve $(M N n, \operatorname{Vgn})$ :

$c:=\operatorname{array}(1 \ldots n, 1 \ldots n):$

for $i$ to $n$ do

for $j$ to $n$ do

$c[i, j]:=\operatorname{evalf}(\ln s[n *(i-1)+j]):$ od: od:

$o p(c)$;

Table of factors $c[i, j]$

\begin{tabular}{rrrrrr}
\hline 0.391733731000 & 0.587600596800 & 0.195866865500 & -0.000000000020 & -0.000000000001 & 0.000000000000 \\
0.367879441200 & 0.551819161800 & 0.183939720600 & 0.000000000000 & 0.000000000000 & 0.000000000000 \\
0.119271450000 & 0.178907174800 & 0.059635725020 & -0.000000000041 & -0.000000000003 & 0.000000000000 \\
0.023485211220 & 0.035227816840 & 0.011742605610 & 0.000000000000 & 0.000000000000 & 0.000000000000 \\
0.003321709383 & 0.004982564074 & 0.001660854692 & 0.000000000000 & 0.000000000000 & 0.000000000000 \\
0.000366528709 & 0.000549793064 & 0.000183264355 & 0.000000000000 & 0.000000000000 & 0.000000000000 \\
\hline
\end{tabular}

$j:={ }^{\prime} j^{\prime}: i:={ }^{\prime}{ }^{\prime}$ :

$f d:=(x, t) \rightarrow \operatorname{sum}(\operatorname{sum}(c[i, j] * u(i, x), i=1 . n) * v(j, t), j=1 . . n):$

$b l:=(x, t) \rightarrow a b s((f(x, t)-f d(x, t)) / f(x, t)):$

tabela: $=\operatorname{array}(-5 . .5,1.5)$ :

for ix from -5 to 5 by 1 do

for it from $I$ to 5 do

tabela $[i x, i t]:=\operatorname{bl}(i x / 5, i t / 5)$ od od:

tab: $=\operatorname{matrix}(11,5,[])$ :

$i:={ }^{\prime} i \cdot: j:=j^{\prime}$ '.

for i from 1 to 11 do

for $j$ from 1 to 5 do

$t a b[i, j]:=$ evalf(tabela $[i-6, j])$ od: od:

$o p(t a b)$;

\begin{tabular}{rccccc}
\hline$x$ & 0.2 & 0.4 & 0.6 & 0.8 & 1 \\
\hline-1.0 & 0.000250936828 & 0.000250934110 & 0.000250933657 & 0.000250933515 & 0.000250933566 \\
-0.8 & 0.000082669943 & 0.000082680932 & 0.000082683173 & 0.000082683714 & 0.000082683964 \\
-0.6 & 0.000026919528 & 0.000026907456 & 0.000026906621 & 0.000026905862 & 0.000026905953 \\
-0.4 & 0.000043402402 & 0.000043393451 & 0.000043391379 & 0.000043390887 & 0.000043390915 \\
-0.2 & 0.000007083831 & 0.000007088716 & 0.000007088886 & 0.000007088907 & 0.000007089022 \\
0 & 0.000030936000 & 0.000030940625 & 0.000030941389 & 0.000030941250 & 0.000030941000 \\
0.2 & 0.000008397107 & 0.000008401713 & 0.000008402679 & 0.000008402864 & 0.000008402634 \\
0.4 & 0.000019343593 & 0.000019341247 & 0.000019340595 & 0.000019340514 & 0.000019340074 \\
0.6 & 0.000010802534 & 0.000010800613 & 0.000010800308 & 0.000010800442 & 0.000010800064 \\
0.8 & 0.000018330937 & 0.000018330094 & 0.000018330001 & 0.000018329813 & 0.000018329926 \\
1 & 0.000039593025 & 0.000039589346 & 0.000039588835 & 0.000039589001 & 0.000039588242 \\
\hline
\end{tabular}




\section{DEPENDENCE OF THE GALERKIN METHOD ON THE NUMBER OF BASIS FUNCTIONS}

Consider the following integral equation in space-time

$$
f(x, t)=x+\sin t-\frac{2}{3} \mathrm{e}^{t}+\frac{2}{3}+\int_{-1}^{1} \int_{0}^{t} y \mathrm{e}^{s} f(y, s) d y d s .
$$

\section{Galerkin method}

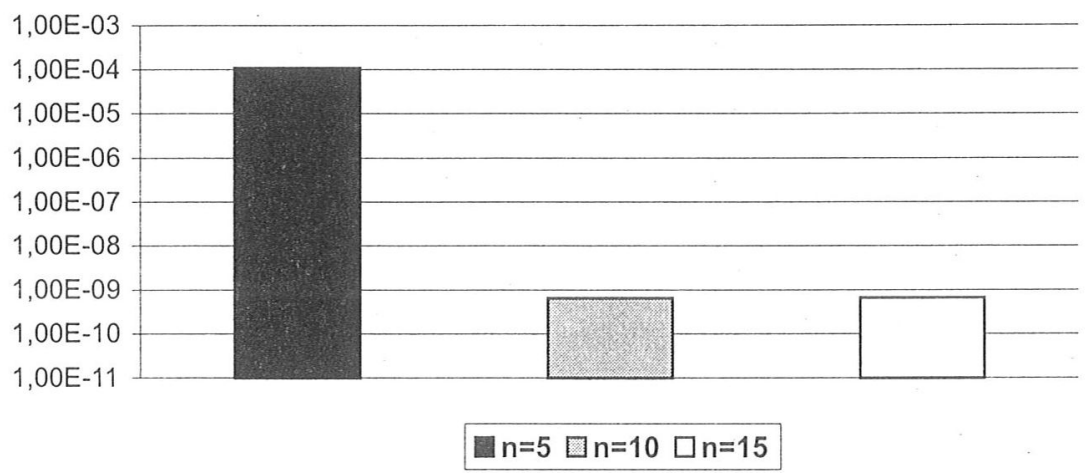

\section{CONCLUSIONS}

In this paper we extend the existing literature related to solving integral equations of the mixed type [1-6], We have shown that increasing the number of the basis functions one obtains better results only for $n \leq 10$; when $n>11$ the Galerkin method becomes weakly stable. Comparing fifteen various numerical examples we conclude that the best results are obtained for $5 \leq n \leq 7$.

\section{References}

[1] H. Brunner, SIAM J. Numer. Anal. 27, 987 (1990).

[2] L. Hącia, Zeit Ang. Math. Mech. 415 (1996).

[3] L. Hącia, Demon. Math. 32. 795 (1999).

[4] J. P. Kauthen, Numer. Math. 56. 409 (1989).

[5] W. I. Tivončuk, Diff. Uravn. 2,91228 (1966) (in Russian).

[6] I. N. Tukalevska, II Sc. Conf. of Young Ukr. Math., Naukova Dumka, Kiev, 609 (1966) (in Ukrainian). 